NBER WORKING PAPER SERIES

\title{
ESTIMATING SELECTION MODELS WITHOUT INSTRUMENT WITH STATA
}

\author{
Xavier D'Haultfoeuille \\ Arnaud Maurel \\ Xiaoyun Qiu \\ Yichong Zhang \\ Working Paper 25823 \\ http://www.nber.org/papers/w25823 \\ NATIONAL BUREAU OF ECONOMIC RESEARCH \\ 1050 Massachusetts Avenue \\ Cambridge, MA 02138 \\ May 2019
}

Zhang acknowledges the financial support from Singapore Ministry of Education Tier 2 grant under grant no. MOE2018-T2-2-169. The views expressed herein are those of the authors and do not necessarily reflect the views of the National Bureau of Economic Research.

NBER working papers are circulated for discussion and comment purposes. They have not been peer-reviewed or been subject to the review by the NBER Board of Directors that accompanies official NBER publications.

(C) 2019 by Xavier D'Haultfoeuille, Arnaud Maurel, Xiaoyun Qiu, and Yichong Zhang. All rights reserved. Short sections of text, not to exceed two paragraphs, may be quoted without explicit permission provided that full credit, including $(\odot$ notice, is given to the source. 
Estimating Selection Models without Instrument with Stata

Xavier D'Haultfoeuille, Arnaud Maurel, Xiaoyun Qiu, and Yichong Zhang

NBER Working Paper No. 25823

May 2019

JEL No. C21,C24,C87,J31

\section{ABSTRACT}

This article presents the eqregsel command for implementing the estimation and bootstrap inference of sample selection models via extremal quantile regression. The command estimates a semiparametric sample selection model without instrument or large support regressor, and outputs the point estimates of the homogenous linear coefficients, their bootstrap standard errors, as well as the p-value for a specification test.

Xavier D'Haultfoeuille

CREST

5 avenue Henry Le Chatelier

91764 Palaiseau cedex

FRANCE

xavier.dhaultfoeuille@ensae.fr

Arnaud Maurel

Department of Economics

Duke University

213 Social Sciences Building

Box 90097

Durham, NC 27708

and NBER

apm16@duke.edu
Xiaoyun Qiu

Toulouse School of Economics

qiu.xyun@gmail.com

Yichong Zhang

School of Economics

Singapore Management University

90 Stamford Rd.

Singapore 178903

Singapore

yczhang@smu.edu.sg 


\title{
Estimating Selection Models without Instrument with Stata*
}

\author{
Xavier D’Haultfouille ${ }^{\dagger} \quad$ Arnaud Maurel $^{\ddagger} \quad$ Xiaoyun Qiu $^{\S} \quad$ Yichong Zhang $^{\llbracket}$
}

May 2019

\begin{abstract}
This article presents the eqregsel command for implementing the estimation and bootstrap inference of sample selection models via extremal quantile regression. The command estimates a semiparametric sample selection model without instrument or large support regressor, and outputs the point estimates of the homogenous linear coefficients, their bootstrap standard errors, as well as the p-value for a specification test.
\end{abstract}

Keywords: eqregsel, sample selection models, extremal quantile regressions.

\section{Introduction}

In this article, we present the command eqregsel for estimation and inference of endogenous sample selection models that implements the procedures developed in recent work by D'Haultfœuille et al. (2018).1 Prior methods proposed in the econometric literature to estimate endogenous sample selection models rely on instruments and/or large support regressors. For the former, see, among others, Heckman (1974, 1979, 1990), Ahn and Powell (1993), Donald (1995), Buchinsky (1998), Chen and Khan (2003), Das et al. (2003), Newey (2009) and Vella (1998) for a survey. Chamberlain (1986) and Lewbel (2007) develop identification strategies for sample selection models in the absence of an instrument for selection. These alternative methods rely on the existence of a large support regressor. However, in practice, valid instruments, as well as large support regressors are often difficult, if not impossible to find.

\footnotetext{
* Zhang acknowledges the financial support from Singapore Ministry of Education Tier 2 grant under grant no. MOE2018-T2-2-169.

${ }^{\dagger}$ CREST-ENSAE. xavier.dhaultfoeuille@ensae.fr.

${ }^{\ddagger}$ Duke University, NBER and IZA. arnaud.maurel@duke.edu.

$\S$ Northwestern University. xiaoyun.qiu@u.northwestern.edu

`Singapore Management University. yczhang@smu.edu.sg.

${ }^{1}$ The Stata command eqregsel can be downloaded from the following webpage: http://www. amaurel. net/Packages.
} 
Instead, the method implemented in eqregsel does not require the presence of instruments or large support regressors ${ }^{2}$ Identification relies instead on the strategy initially proposed by D'Haultfoeuille and Maurel (2013), which is based on the idea that, provided that selection is endogenous, one can expect the effect of the outcome on selection to dominate those of the covariates, for large values of the outcome. eqregsel builds on the estimation method proposed by D'Haultfouille et al. (2018) and implements a series of quantile regressions in the tails of the outcome distribution (extremal quantile regressions) ${ }^{3}$ The command outputs estimates for a set of user-specified coefficients of interest, their standard errors (estimated via bootstrap), and a p-value for the specification test described in D'Haultfœuille et al. (2018). Our command complements the existing Stata command heckman for the estimation of sample selection models. In terms of underlying assumptions, eqregsel has at least three distinctive features compared to heckman. First, it does not require normality of the error term in the selection equation, nor linearity of the conditional expectation of the error term in the outcome equations. Second, it does not restrict the selection process, apart from an independence at infinity condition. Third, it allows for heterogeneous distributional effects of other control variables.

The remainder of the paper is organized as follows. In Section 2 , we recall the setup of the semiparametric endogenous sample selection model considered in D'Haultfœuille et al. (2018), and describe the data-driven procedure used to choose the quantile index for the extremal quantile regression. Section 3 describes how to implement the method in practice. Section 4 presents the eqregsel command. Section 5 illustrates the use of our command by estimating the black-white wage gap on US young males of the 1979 and 1997 National Longitudinal Surveys of Youth. Section 6 concludes.

\section{The framework and estimation method}

\subsection{Model and estimation}

We consider the following outcome equation:

$$
Y^{*}=X_{1}^{\prime} \beta_{1}+\varepsilon
$$

\footnotetext{
${ }^{2}$ See Honoré and $\mathrm{Hu}(2018)$ for a related recent work, also motivated by the difficulty of finding instruments for sample selection. As is the case here, they do not require exclusion restrictions nor large support regressors. However, their approach is based on a different set of assumptions and, in contrast to our framework, delivers set- rather than point-identification.

${ }^{3}$ See Chernozhukov et al. (2017) for an overview of extremal quantile regression methods and recent applications.
} 
where $Y^{*} \in \mathbb{R}$ and $X_{1} \in \mathbb{R}^{d_{1}}$ are the outcome and covariates of interest, respectively. In the following, we seek to identify and estimate $\beta_{1}$. For that purpose, we rely on two key conditions. The first is that for any $\tau \in(0,1)$, the $\tau$-th conditional quantile of $\varepsilon$ satisfies

$$
Q_{\varepsilon \mid X}(\tau \mid X)=\beta_{0}(\tau)+X_{2}^{\prime} \beta_{2}(\tau)
$$

where $X=\left(X_{1}^{\prime}, X_{2}^{\prime}\right)^{\prime}$ and $X_{2}$ denotes other covariates. Then

$$
Q_{Y^{*} \mid X}(\tau)=X_{1}^{\prime} \beta_{1}+\beta_{0}+X_{2}^{\prime} \beta_{2}(\tau)
$$

The effect of $X_{1}$ is thus assumed to be homogenous across different quantile indices, while the effect of the other covariates $X_{2}$ is allowed to be heterogeneous across the distribution of $Y^{*}$. $Y^{*}$ is not directly observed. Instead, and denoting by $D$ the selection dummy, the econometrician only observes $D, Y=D Y^{*}$ and $X$. The second key condition is that conditional on having "large" outcomes, selection is independent of the covariates. More precisely, we assume that there exists a constant $h \in(0,1]$ such that for all $x \in \operatorname{Supp}(X)$,

$$
\lim _{y \rightarrow \infty} P\left(D=1 \mid X=x, Y^{*}=y\right)=h .
$$

Combining (2.2) and (2.3), D'Haultfœuille et al. (2018, Theorem 1) shows that, under some regularity conditions on the upper tail of $\varepsilon$, as $\tau \rightarrow 0$,

$$
\begin{aligned}
Q_{-Y \mid X}(\tau \mid X) & =Q_{-Y^{*} \mid X}(\tau / h \mid X)+o(1) \\
& =-X_{1}^{\prime} \beta_{1}-\beta_{0}(1-\tau / h)-X_{2}^{\prime} \beta_{2}(1-\tau / h)+o(1) .
\end{aligned}
$$

Therefore, 2.4 suggests that we can estimate $\beta_{1}$ by running a quantile regression of $-Y$ on $-X$ with a sufficiently small quantile index $\tau$, i.e.,

$$
\left(\widehat{\beta}_{1}^{\prime}, \widehat{\beta}_{0}(1-\tau / h), \widehat{\beta}_{2}^{\prime}(1-\tau / h)\right)^{\prime}=\arg \min _{\beta} \sum_{i=1}^{n} \rho_{\tau}\left(-Y_{i}+\bar{X}_{i}^{\prime} \beta\right),
$$

where $\rho_{\tau}(u)=(\tau-\mathbb{1}\{u<0\}) u$ is the check function used in quantile regressions and $\bar{X}_{i}=$ $\left(X_{1 i}^{\prime}, 1, X_{2 i}^{\prime}\right)^{\prime}$. Intuitively, for $\widehat{\beta}_{1}$ to be consistent, $\tau$ should depend on $n$ and tend to 0 as $n$ tends to infinity. However, it should not tend too quickly to 0 , otherwise the extremal quantile regression would be unstable. Formally, and letting $\tau_{n}$ denote the quantile index, D'Haultfœuille et al. (2018) establish that if $\tau_{n} \rightarrow 0$ and $n \tau_{n} \rightarrow \infty, 4$, and under additional

\footnotetext{
${ }^{4}$ This corresponds to the so-called "intermediate order case" in extreme value theory, in contrast to "extreme order cases" where one would have $n \tau_{n} \rightarrow k$ for some $k>0$.
} 
technical restrictions, $\widehat{\beta}_{1}$ is consistent and asymptotically normal.

As is standard with extremal quantile regressions (see Chernozhukov et al., 2017), the rate of convergence is not the usual parametric root-n rate. Moreover, in this case, this rate depends on unknown features of the distribution of $\left(D, Y^{*}, X\right) 5^{5}$ Importantly, D'Haultfouille et al. (2018) show that the bootstrap is consistent for inference, and does not require the knowledge of the rate of convergence. To illustrate this, let $q_{\gamma}^{*}$ denote the quantile of order $\gamma$ of the bootstrap estimator $\widehat{\beta}_{1}^{*}$, assuming for simplicity that $X_{1}$ is a scalar $\left(d_{1}=1\right)$. Then, Theorem 2 in D'Haultfœuille et al. (2018) implies that the percentile bootstrap confidence interval $\left[q_{\alpha / 2}^{*}, q_{1-\alpha / 2}^{*}\right]$ of $\beta_{1}$ has an asymptotic coverage of $1-\alpha$. Such an interval does not require the knowledge of the rate of convergence.

The results above rely on two main conditions, namely (2.1) and 2.3. Importantly, we can develop a specification test of these conditions, based on the implication that the coefficient $\beta_{1}$ in $Q_{-Y \mid X}\left(\tau_{n} \mid X\right)$ is the same across different extremal quantile indices $\tau_{n}$ (see (2.4)). Then, if the model is correctly specified, the two estimators $\widehat{\beta}_{1}\left(\ell \tau_{n}\right)$ (with $0<\ell<1$ ) and $\widehat{\beta}_{1}\left(\tau_{n}\right)$ of $\beta_{1}$, obtained respectively with $\tau=\ell \tau_{n}$ and $\tau=\tau_{n}$, should be close. Following this idea, consider the following J-test statistic:

$$
T_{J}(\ell)=[(1 / \ell)-1]^{2}\left(\widehat{\beta}_{1}\left(\tau_{n}\right)-\widehat{\beta}_{1}\left(\ell \tau_{n}\right)\right)^{\prime} \widehat{\Omega}^{-1}\left(\widehat{\beta}_{1}\left(\tau_{n}\right)-\widehat{\beta}_{1}\left(\ell \tau_{n}\right)\right)
$$

where $\widehat{\Omega}$ is a (bootstrap) estimator of the asymptotic covariance of $\widehat{\beta}_{1}\left(\tau_{n}\right)$, properly normalized by the rate of convergence in view of the discussion above. Then we reject the test at the nominal level $\alpha$ whenever $T_{J}(\ell)>q_{d_{1}}(1-\alpha)$, where $q_{d_{1}}(1-\alpha)$ is the quantile of order $1-\alpha$ of a $\chi^{2}$ distribution with $d_{1}$ degrees of freedom. Theorem 2.3 in D'Haultfœuille et al. (2018) establishes that for any $0<\ell<1$ the test has an asymptotic level of $\alpha$. It also proves that under some local alternatives, the local power is maximized at $\ell^{*}=\arg \max _{\ell \in[0,1]} \ell[\ln (l)]^{2} /(1-$ $\ell) \simeq 0.2$.

\subsection{Choice of the quantile index}

The performance of extremal quantile estimators depends on a trade-off between bias and variance, which is governed by the quantile index $\tau_{n}$ used in the extremal quantile regression. We present in the following the algorithm outlined in D'Haultfœuille et al. (2018), which selects a suitable quantile index based on estimators of the bias and the variance of $\widehat{\beta}_{1}$.

Specifically, consider the same test statistic as in (2.6), but where $\left(\ell \tau_{n}, \tau_{n}\right)$ are replaced by

\footnotetext{
${ }^{5}$ We refer to the definition of the rate above Theorem 2.2 in D'Haultfouille et al. (2018) for more details.
} 
$\left(\ell_{1} \tau_{n}, \ell_{2} \tau_{n}\right)$, with $\ell_{1}<1<\ell_{2}$ :

$$
T_{J}(\tau)=\left[1 / \ell_{1}-1 / \ell_{2}\right]^{2}\left(\widehat{\beta}_{1}\left(\ell_{2} \tau\right)-\widehat{\beta}_{1}\left(\ell_{1} \tau\right)\right)^{\prime} \widehat{\Omega}^{-1}\left(\widehat{\beta}_{1}\left(\ell_{2} \tau\right)-\widehat{\beta}_{1}\left(\ell_{1} \tau\right)\right)
$$

D'Haultfœuille et al. (2018) shows that the difference between the median of $T_{J}(\tau)$ and the median of a chi-squared distribution with $d_{1}$ degrees of freedom can serve as a proxy for the bias of the estimator.

The idea, then, is to estimate this difference using subsampling 6 For each subsample and each quantile index $\tau$ within a grid $\mathcal{G}$, one can compute $T_{J}(\tau)$. Let $M_{\text {sub }}(\tau)$ denote the median of these test statistics over different subsamples for a given $\tau$, and let $M_{d_{1}}$ denote the median of the chi-squared distribution with $d_{1}$ degrees of freedom. Then, the proxy of the bias is defined as

$$
\widehat{\operatorname{diff}}_{n}(\tau)=\frac{\left|M_{\mathrm{sub}}(\tau)-M_{d_{1}}\right|}{\sqrt{b_{n} \tau}}
$$

where $b_{n}$ denotes the subsample size.

Similarly, the asymptotic covariance matrix is estimated by the covariance matrix of the subsampling estimator of $\beta_{1}$, multiplied by the normalizing factor $b_{n} / n$. Denote by $\widehat{\operatorname{Var}}_{n}(\tau)$ the sum of the diagonal elements of this covariance matrix. The quantile index is selected to optimize the bias-variance trade-off:

$$
\widehat{\tau}_{n}=\arg \min _{\tau \in \mathcal{G}} \widehat{\operatorname{Var}}_{n}(\tau)+\widehat{\operatorname{diff}}_{n}(\tau)
$$

where $\mathcal{G}$ denotes a finite grid within $(0,1)$. This procedure results in undersmoothing in comparison with a more standard trade-off between variance and squared bias. Similarly to the case of nonparametric regressions, this is needed to control the asymptotic bias that would otherwise affect the limiting distribution of the estimator. We refer to D'Haultfœuille et al. (2018) for simulation-based evidence that this choice leads to estimators that are both accurate and only very mildly biased, thus leading to reliable inference on $\beta_{1}$.

\section{Implementation}

We summarize how we implement the method described above in eqregsel.

1. Draw $B$ bootstrap samples and $B$ subsamples of size $b_{n}$.

\footnotetext{
${ }^{6}$ We recall that subsampling corresponds to a bootstrap without replacement of size $b_{n}<n$. Though often less accurate than the standard bootstrap, subsampling has the advantage of being consistent under much weaker conditions. See Politis et al. (1999) for an introduction.
} 
2. For each $\tau \in \mathcal{G}$ :

(a) Compute the estimator of $\beta(\tau)=\left(\beta_{1}^{\prime}, \beta_{0}(1-\tau / h), \beta_{2}^{\prime}(1-\tau / h)\right)^{\prime}$ :

$$
\widehat{\beta}(\tau)=\arg \min _{\beta} \sum_{i=1}^{n} \rho_{\tau}\left(-Y_{i}+\bar{X}_{i}^{\prime} \beta\right) .
$$

Let $\widehat{\beta}_{1}(\tau)$ denote the vector comprising the first $d_{1}$ components of $\widehat{\beta}(\tau)$.

(b) Compute

$$
\widehat{\Omega}(\tau)=\frac{1}{B} \sum_{b=1}^{B}\left(\widehat{\beta}_{1}^{b}(\tau)-\widehat{\beta}_{1}(\tau)\right)\left(\widehat{\beta}_{1}^{b}(\tau)-\widehat{\beta}_{1}(\tau)\right)^{\prime}
$$

with $\widehat{\beta}_{1}^{b}(\tau)$ the bootstrap estimator of $\beta_{1}$ on the $b$-th bootstrap sample.

(c) Compute, for each subsample $s=1 \ldots B$, the estimator of $\beta_{1}\left(\widehat{\beta}_{1}^{s}(\tau)\right)$, and the J-test statistic 7

$$
T_{J}^{s}(\tau)=\left(b_{n} / n\right)\left[1 / \ell_{1}-1 / \ell_{2}\right]^{2}\left(\widehat{\beta}_{1}^{s}\left(\ell_{2} \tau\right)-\widehat{\beta}_{1}^{s}\left(\ell_{1} \tau\right)\right)^{\prime} \widehat{\Omega}^{-1}(\tau)\left(\widehat{\beta}_{1}^{s}\left(\ell_{2} \tau\right)-\widehat{\beta}_{1}^{s}\left(\ell_{1} \tau\right)\right)
$$

(d) Compute $\widehat{\operatorname{diff}}_{n}(\tau)=\frac{\left|M_{\mathrm{sub}}(\tau)-M_{d_{1}}\right|}{\sqrt{b_{n} \tau}}$ where $M_{\mathrm{sub}}(\tau)$ denotes the median of $\left(T_{J}^{1}(\tau), \ldots, T_{J}^{B}(\tau)\right)$.

(e) Compute $\widehat{\operatorname{Var}}_{n}(\tau)=\left(b_{n} / n\right) \sum_{k=1}^{d_{1}} \widehat{\Sigma}(\tau)_{k k}$, where $\widehat{\Sigma}(\tau)_{k k}$ is the $k$-th diagonal term of

$$
\widehat{\Sigma}(\tau)=\frac{1}{B} \sum_{s=1}^{B}\left(\widehat{\beta}_{1}^{s}(\tau)-\bar{\beta}_{1}(\tau)\right)\left(\widehat{\beta}_{1}^{s}(\tau)-\bar{\beta}_{1}(\tau)\right)^{\prime} \quad \text { with } \quad \bar{\beta}_{1}(\tau)=\frac{1}{B} \sum_{s=1}^{B} \widehat{\beta}_{1}^{s}(\tau)
$$

3. Compute $\widehat{\tau}_{n}=\arg \min _{\tau \in \mathcal{G}} \widehat{\operatorname{Var}}_{n}(\tau)+\widehat{\operatorname{diff}}_{n}(\tau)$.

4. Define $\widehat{\beta}_{1}=\widehat{\beta}_{1}\left(\widehat{\tau}_{n}\right)$ and $\widehat{\Omega}=\widehat{\Omega}\left(\widehat{\tau}_{n}\right)$. Confidence intervals $\mathrm{CI}_{1-\alpha}\left(\beta_{1 k}\right)$ of level $1-\alpha$ on the $k$-th component of $\beta_{1}$ are then equal to

$$
\mathrm{CI}_{1-\alpha}\left(\beta_{1 k}\right)=\left[\widehat{\beta}_{1 k}-z_{1-\alpha / 2} \sqrt{\widehat{\Omega}_{k k}}, \widehat{\beta}_{1 k}+z_{1-\alpha / 2} \sqrt{\widehat{\Omega}_{k k}}\right]
$$

where $\widehat{\Omega}_{k k}$ is the $k$-th diagonal term of $\widehat{\Omega}$ and $z_{1-\alpha / 2}$ is the quantile of order $1-\alpha / 2$ of a standard normal variable.

5. Compute $\widehat{\beta}_{1}\left(0.2 \widehat{\tau}_{n}\right)$ and then $T_{J}(0.2)$, as defined in $(2.6)$, to perform the specification test of the model.

\footnotetext{
${ }^{7}$ The term $b_{n} / n$ accounts for the fact that the rate of convergence of the $J$ statistic on the subsample is $b_{n} / n$ times the rate of convergence on the whole sample.
} 
In practice, we consider an equally-spaced grid $\mathcal{G}$ with lower bound $\min \left(0.1,80 / b_{n}\right)$, upper bound 0.3 and a number of points equal to $n_{\mathcal{G}}$. The lower bound is motivated by the fact that if the effective subsampling size $\tau b_{n}$ becomes too small, then the intermediate order asymptotic theory is likely to be a poor approximation (see Chernozhukov and Fernandez-Val, 2011 for a related discussion). To compute $T_{J}^{s}(\tau)$ in Step 2.(c) above, we use $\left(\ell_{1}, \ell_{2}\right)=(0.9,1.1)$.

\section{The eqregsel command}

We describe below the syntax, options and saved results associated with the eqregsel command. Note that it relies on the moremata Stata package. If the latter is not already installed, one must type ssc install moremata in Stata command line. The eqregsel command is compatible with Stata 14 and later versions.

\subsection{Syntax}

The syntax of eqregsel is as follows:

$\underline{\text { eqregsel }}$ Y X1 X2 $[$ if $][i n][, \operatorname{hom}(\#) \operatorname{subs}(\#) \operatorname{grid}(\#) \operatorname{rep}(\#)]$

\subsection{Description}

eqregsel computes $\widehat{\beta}_{1}$ in 2.2 based on the data-driven $\tau_{n}$ detailed in Section 2.2 above. It also reports its standard errors and 95\% confidence intervals. Finally, it computes the p-value of this specification test using $\ell=0.2$.

$\mathrm{X} 1$ is the list of variable entering in $X_{1}$ in Model (2.2).

$\mathrm{X} 2$ is the list of variable entering in $X_{2}$ in Model (2.2).

\subsection{Options}

hom(\#) specifies $d_{1}$, the number of variables in $X_{1}$. The code then returns their estimated effects and standard errors. The default value is 1 .

$\operatorname{subs}(\#)$ specifies the subsample size $b_{n}$. Following D'Haultfœuille et al. (2018), and letting $x^{+}=\max (0, x)$, the default value is set to

$$
b_{n}=0.6 n-0.2(n-500)^{+}-0.2(n-1000)^{+}-0.2\left[1-\frac{\ln (2000)}{\ln (n)}\right](n-2000)^{+} .
$$

$\operatorname{grid}(\#)$ specifies $n_{\mathcal{G}}$, the number of grid points. The default value is 40 . 
rep (\#) specifies $B$, the number of bootstrap and subsampling replications. The default value is 150 .

\subsection{Saved results}

The eqregsel command saves the following in e():

1. e (tau0), a scalar containing the quantile index $\widehat{\tau}_{n}$.

2. e(specificationtest), a scalar containing the p-value of the specification test.

3. e (subs), a scalar containing the subsample size $b_{n}$.

4. e (homvar), a scalar containing $d_{1}$, the number of variable(s) with homogenous effect(s) on the outcome.

5. e (beta_hom), a $d_{1} \times 1$ matrix containing the estimated coefficient(s) of interest.

6. e (std_b), a $d_{1} \times 1$ matrix containing the standard error of the estimator(s).

\section{Example}

We use the command eqregsel to estimate the black-white wage gap among young males from the National Longitudinal Surveys of Youth 1979 and 1997 (NLSY79 and NLSY97), revisiting the work of D'Haultfœuille et al. (2018) on this question. We are in particular interested in the evolution of the gap between these two cohorts. We use the same samples and definitions of variables as in D'Haultfœuille et al. (2018). In particular, we consider that an individual in the NLSY79 is a nonparticipant if he did not work in 1990 nor in 1991 . The outcome of interest is the (potential) log-wage, which is defined as the log of the mean real wages in 1990 and 1991 for workers who worked both years, and the log of the real wage in the year of employment for those who worked only one year. We apply the same rules with the years 2007 and 2008 for individuals in the NLSY97.

In our specification, we estimate for the two samples the effect of the Black dummy on the log of wages (log_wage), controlling for Hispanic dummy (hispanic), age (age), AFQT (Armed Forces Qualification Test score) and AFQT squared (afqt and afqt2). The AFQT scores cannot be directly compared across both NLSY cohorts, in part because of changes in how the test was administered. To handle this issue, we use a modified version of the AFQT constructed using the equipercentile mapping proposed by Altonji et al. (2012). We also restrict the samples to the respondents who took the test when they were 16 or 17 , to address 
the issue that the rank within the AFQT distribution may vary with the age of the respondent at the time of the test. The final sample sizes are equal to 1,077 and 1,123 for the NLSY79 and NLSY97 cohorts, respectively. The overall labor force participation rates for the two corresponding samples are equal to $95.1 \%$ and $89.7 \%$. They only reach $90.6 \%$ and $81.4 \%$ for Black males, however.

We report below the output of the eqregsel procedure applied to the NLSY79 and NLSY97 samples, respectively. We use the default parameters. We can see from the estimation output that the default subsample sizes used in bootstrapping are 515 and 524, given the total sample size of 1,077 and 1,123. The procedure also displays the estimated computing time, along with a progress bar. Although in this example estimation is performed at a limited computational cost, this feature makes it possible for the user to stop the execution of the command. If needed, one can then save on execution time by setting a lower number of bootstrap and subsampling replications, or a lower number of grid points 8

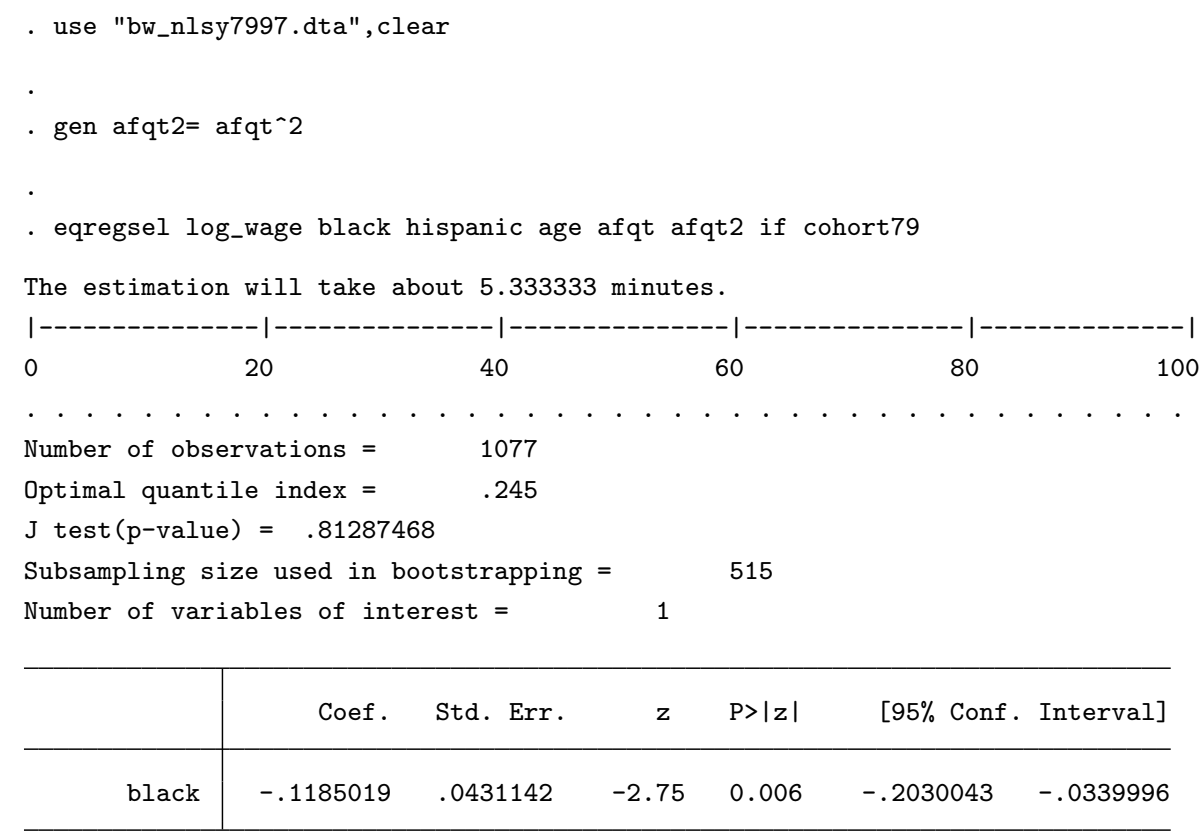

\footnotetext{
${ }^{8}$ The computation times reported in these examples are obtained on an Intel Xeon CPU 2.40 GHz processor with 128 GB of RAM, using Stata MP 14.2.
} 


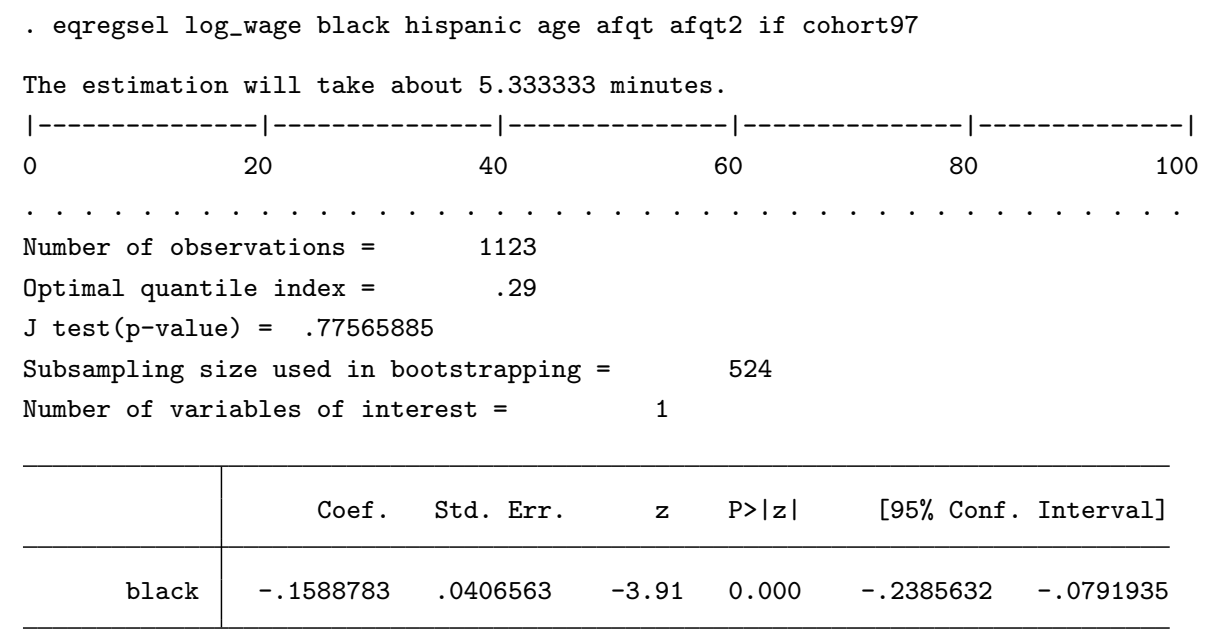

The estimation results point to statistically as well as economically significant black-white wage gaps for the two cohorts. We also observe a wider black-white wage gap for the 1997 cohort relative to the 1979 cohort, with an increase in the estimated gap from about $11.9 \%$ to $15.9 \%$. Note, however, that the difference is not significant at usual levels ( $\mathrm{p}$-value=0.51). Interestingly, the p-values of the specification tests imply that one cannot reject our specification for either cohort at any standard statistical level.

It is interesting to compare the estimated black-white wage gap with the results of a simple OLS regression of the log of hourly wages on a black dummy and the same set of controls. The estimated black-white wage gap drops from $11.9 \%$ and $15.9 \%$, for our specifications, to $8.1 \%$ and $9.7 \%$ (with standard errors equal to 0.035 and 0.041 ), for the OLS specification that ignores selection. That the estimated wage gap is larger in magnitude when we use our method is consistent with the underlying sample selection issue. Indeed, among males, blacks are significantly more likely to dropout from the labor market (Juhn, 2003). Since dropouts tend to have lower potential wages, one can expect that not controlling for endogenous labor market participation will result in underestimating the black-white wage differential.99

\section{Conclusion}

In this paper we have discussed how to use the eqregsel command to estimate and conduct inference on sample selection models, following D'Haultfouille et al. (2018). Unlike alternative

\footnotetext{
${ }^{9}$ We also estimate the wage gap using the Heckman two-step estimator, without any instrument. We obtain very imprecisely estimated gaps of $24.2 \%$ and $-21.2 \%$, with standard errors of 0.48 and 0.68 . This could be expected: in the absence of instrument, this estimator strongly relies on functional form restrictions and is often unstable.
} 
estimation methods that have been proposed in the literature, the method does not require the presence of instruments or large support regressors. The estimator is simply based on a quantile regression in the tail, but with a quantile index chosen in a data-driven fashion. The Stata command eqregsel makes it possible to easily use this procedure. 


\section{References}

Ahn, H., and J. L. Powell. 1993. Semiparametric estimation of censored selection models with a nonparametric selection mechanism. Journal of Econometrics 58(1-2): 3-29.

Altonji, J., P. Bharadwaj, and F. Lange. 2012. Changes in the characteristics of American youth: Implications for adult outcomes. Journal of Labor Economics 30(4): 783-828.

Buchinsky, M. 1998. The dynamics of changes in the female wage distribution in the USA: A quantile regression approach. Journal of Applied Econometrics 13(1): 1-30.

Chamberlain, G. 1986. Asymptotic efficiency in semiparametric model with censoring. Journal of Econometrics 32(2): 189-218.

Chen, S., and S. Khan. 2003. Semiparametric estimation of a heteroskedastic sample selection model. Econometric Theory 19(6): 1040-1064.

Chernozhukov, V., and I. Fernandez-Val. 2011. Inference for extremal conditional quantile models, with an application to market and birthweight risks. Review of Economic Studies 78(2): 559-589.

Chernozhukov, V., I. Fernandez-Val, and T. Kaji. 2017. Extremal quantile regression: An overview. In Handbook of Quantile Regression, ed. R. Koenker, V. Chernozhukov, X. He, and L. Peng. Chapman and Hall/CRC.

Das, M., W. Newey, and F. Vella. 2003. Nonparametric estimation of sample selection models. Review of Economic Studies 70(1): 33-58.

D'Haultfoeuille, X., and A. Maurel. 2013. Another look at the identification at infinity of sample selection models. Econometric Theory 29(1): 213-224.

D'Haultfœuille, X., A. Maurel, and Y. Zhang. 2018. Extremal quantile regressions for selection models and the black-white wage gap. Journal of Econometrics 203(1): 129-142.

Donald, S. 1995. Two-step estimation of heteroskedastic sample selection models. Journal of Econometrics 65(2): 347-380.

Heckman, J. J. 1974. Shadow prices, market wages, and labor supply. Econometrica 42(4): 679-694.

1979. Sample selection bias as a specification error. Econometrica 47(1): 153-161. 
—. 1990. Varieties of selection bias. The American Economic Review 80(2): 313-318.

Honoré, B., and L. Hu. 2018. Selection without exclusion. Working paper.

Juhn, C. 2003. Labor market dropouts and trends in the wages of black and white men. Industrial and Labor Relations Review 56(4): 643-662.

Lewbel, A. 2007. Endogenous selection or treatment model estimation. Journal of Econometrics 141(2): 777-806.

Newey, W. 2009. Two step series estimation of sample selection models. The Econometrics Journal 12(1): 217-229.

Politis, D. N., J. P. Romano, and M. Wolf. 1999. Subsampling. Springer Science \& Business Media.

Vella, F. 1998. Estimating models with sample selection bias: A survey. Journal of Human Resources 33(1): 127-169. 